Bull. Mater. Sci., Vol. 3, Number 2, July 1981 pp. 119-131. (C) Printed in India.

\title{
Statistical theories of nematic liquid crystals
}

\author{
N V MADHUSUDANA \\ Raman Research Institute, Bangalore 560 080, India \\ MS received 26 January 1981
}

\begin{abstract}
Nematic liquid crystals are composed of molecules with moderate shape anisotropy, and are characterized by purely orientational but no translational long range order. It is necessary to take into account both the anisotropic attractions between the molecules and the excluded volume effects to give a proper statistical theory of this phase. Indeed a reasonably satisfactory semiquantitative description has emerged only in the past two years. The paper reviews recent developments in the field.
\end{abstract}

Keywords. Nematic liquid erystals; statistical theory.

\section{Introduction}

An essential requirement for the formation of thermotropic liquid crystals is shape anisotropy of the molecules, with the ratio of the largest dimension to the shortest dimension generally in the range of 3-10 (for an introduction see Chandrasekhar 1977). The vast majority of mesogens are rod-like but it was shown for the first time by Chandrasekhar et al (1977) that relatively simple pure disc-shaped compounds also exhibit liquid crystalline phases.

Nematics are the simplest type of liquid crystals, and are characterized by only a long range orientational order, but no long range translational order of the molecules (figure 1). As a consequence, this phase (and in particular the nematic-isotropic

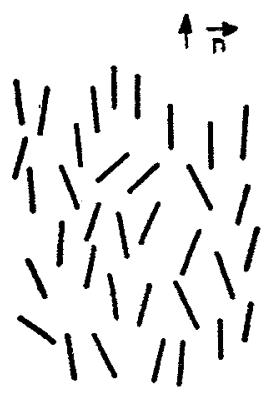

Figure 1. Schematic diagram of the arrangement of rod-like molecules in the orientationally ordered nematic liquid crystals. $\vec{n}$ is the direction of average orientation of the molecules. 
hexa pentyloxy benzoate of triphenylene

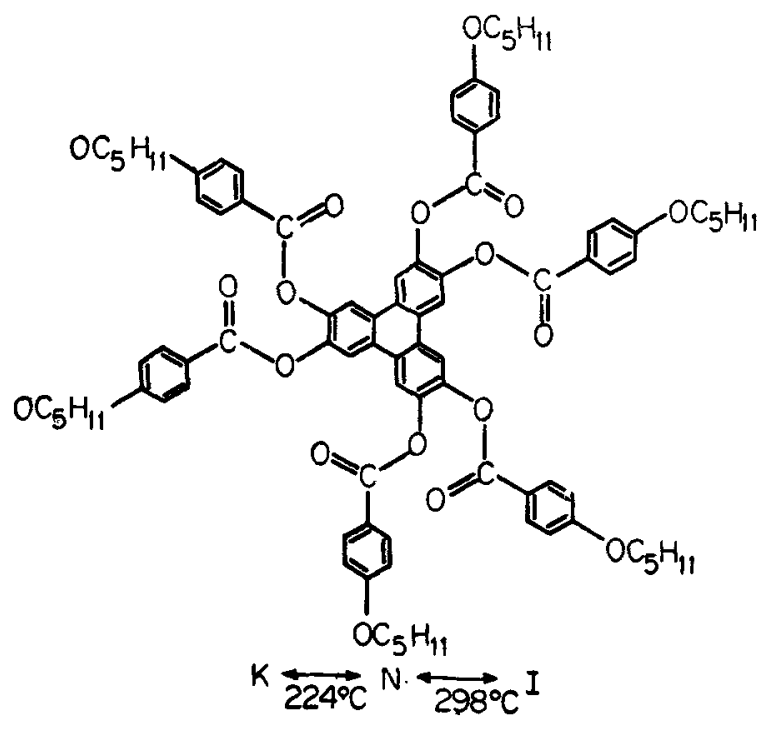

p-Azoxyanisole (PAA)<smiles>COc1ccc(N=Nc2ccc(OC)cc2)cc1</smiles>

$K \frac{118.2^{\circ} \mathrm{C}}{(29.6 \mathrm{~kJ})} N \frac{135 \cdot 3^{\circ} \mathrm{C}}{(0.6 \mathrm{~kJ})} \mathrm{I}$

$p$-Quinquephenyl

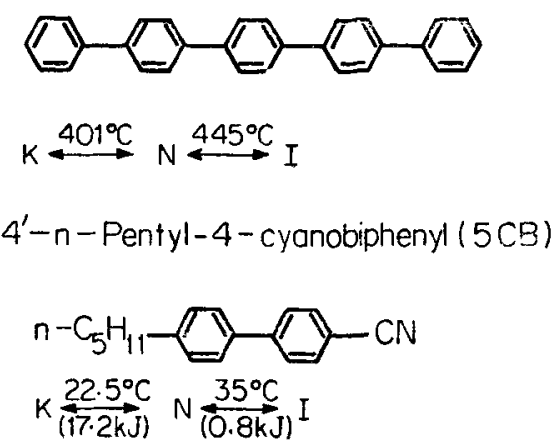

Figure 2. Molecular structures of a few compounds exhibiting the nematic phase. The figures in brackets in some cases indicate the molar heats of transition. Quinquephenyl has no net dipole moment while 5CB has a strong dipole moment. The figure on the left side corresponds to a disc-like nematogen (Destrade et al 1980).

phase transition) has attracted a large number of theoretical studies (see Chandrasekhar and Madhusudana 1978). We will briefly review the more important of these studies, highlighting the most recent developments.

Both rod-shaped and disc-shaped molecules exhibit the nematic phase (see figure 2 for some examples). We define a unit vector $\vec{n}$ called the director to denote the direction of average orientation of the molecules. Experiments indicate that the properties of the medium do not depend on the sign of $\vec{n}$. In such a case, if the molecules are assumed to be cylindrically symmetric, the order parameter can be simply defined as

$$
s=\left\langle\frac{3 \cos ^{2} \theta-1}{2}\right\rangle \text {, }
$$

where $\theta$ is the angle that the cylinder axis makes with $\vec{n}$. $s$ can be determined by a variety of techniques like NMR, optical birefringence, etc. It is found that $s$ decreases with increase of temperature, dropping abruptly from a finite value $s_{c}$ to zero in a weak first order nematic-isotropic transition at a temperature $T_{N I}$ (figure 3 ). Properties like the coefficient of thermal expansion $\alpha$, specific heat $\left(C_{v}\right.$ and $\left.C_{p}\right)$, etc exhibit strong pre- and post-transition effects close to $T_{N I}$.

\section{Theories based on anisotropic attractive potentials}

Historically, the first molecular theory of liquid crystals was proposed by Born (1916). He assumed that interactions between strong dipole moments associated with the 


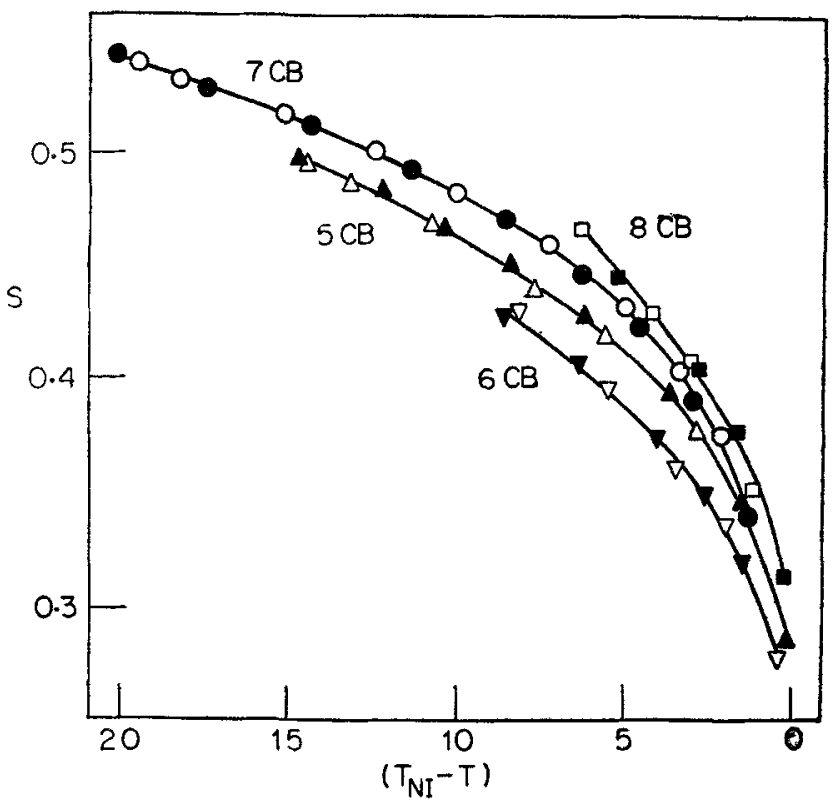

Figure 3. Variation of the orientational order parameters in the nematic phase of several homologues of $5 \mathrm{CB}$ (see figure 2 ) as functions of relative temperature (Karat and Madhusudana 1976).

molecules lead to the orientational order and that the nematic liquid crystal is a ferroelectric. This is an incorrect picture, in view of what has been said earlier, namely that the director is apolar.

Maier and Saupe (1959) developed a mean field theory of nematics by assuming that anisotropic dispcrsion interactions between the molecules are responsible for the stability of the phase. The orientational energy of a molecule $i$ is given by

$$
u_{i}=-\frac{A}{V^{2}} s P_{2}\left(\cos \theta_{i}\right)
$$

where $A$ is a constant depending on the molecular species, $V$ the molar volume and $P_{2}\left(\cos \theta_{i}\right)$ the second Legendre polynomial. The molar free energy is then given by

$$
\begin{aligned}
F=N k_{B} T & {\left[\frac{A}{2 k_{B} T V^{2}} s(s+1)\right.} \\
& \left.-\ln \int_{0}^{1} \exp \left(\frac{3 A}{2 k_{B} T V^{2}} s \cos ^{2} \theta_{i}\right) d\left(\cos \theta_{i}\right)\right],
\end{aligned}
$$

where $N$ is the Avogadro number and $k_{B}$ the Boltzmann constant. The free energy is minimized by the consistency condition

$$
s=\left\langle\frac{3 \cos ^{2} \theta_{i}-1}{2}\right\rangle
$$


where the averaging is done using a Boltzmann factor corresponding to the potential energy (2). The calculations lead to a first order NI transition at $s_{c} \simeq 0.42$, a universal value. Experimentally, however, one finds that $s_{c}$ varies between 0.3 to 0.5 for different compounds. Higher order terms can be introduced in the potential (2) to account for these differences (Chandrasekhar and Madhusudana 1971). But the mean field theories overestimate the strength of the transition. Further, $\left(T_{N I}-T^{*}\right)$, where $T^{*}$ is a hypothetical second order NI transition temperature (de Gennes 1971) is predicted to be $\simeq 30-40^{\circ}$ whereas the experimental value obtained for instance by Cotton-Mouton constants of the isotropic phase is $\simeq 1-2^{\circ}$.

Obviously, near-neighbour correlations or short-range order effects have to be taken into account to improve the theory. A Bethe-type cluster is used in such calculations (Madhusudana and Chandrasekhar 1973a). Each molecule is assumed to have $z$ nearest neighbours $(z \geqslant 3)$, no two nearest neighbours being considercd to be nearest neighbours of each other. Let $E\left(\theta_{0 j}\right)$ be the pair potential between the central molecule ' 0 ' and one of its neighbours $j, \theta_{0 j}$ being the angle between them. Each outer shell molecule is subject to an average potential $V\left(\theta_{j}\right)$ due to the rest of the medium. The relative weight for a given configuration of a cluster of $(z+1)$ molecules is then

$$
\prod_{i=1}^{z} f\left(\theta_{0 j}\right) g\left(\theta_{j}\right)
$$

where $f\left(\theta_{0 j}\right)=\exp \left[-E\left(\theta_{0 j}\right) / k_{B} T\right]$,

and $\quad g\left(\theta_{j}\right)=\exp \left[-V\left(\theta_{j}\right) / k_{B} T\right]$.

The relative probability that the central molecule has the spherical angles $\left(\theta_{0}, \phi_{0}\right)$ is given by

$$
\int \cdots \int \prod_{i=1}^{z} f\left(\theta_{0 j}\right) g\left(\theta_{j}\right) d \phi_{j}
$$

while that for an outer molecule, say 1 , to assume an orientation $\left(\theta_{1}, \phi_{1}\right)$ is

$$
\iint f\left(\theta_{01}\right) g\left(\theta_{1}\right) d\left(\cos \theta_{0}\right) d \phi_{0} \int \ldots \int \prod_{=2}^{z} f\left(\theta_{0 j}\right) g\left(\theta_{j}\right) d\left(\cos \theta_{j}\right) d \phi_{j}
$$

Chang (1937) postulated that for consistency of description, the two probabilities are identical when the central and outer molecules assume the same orientation. There are other equivalent ways of expressing this consistency condition (Krieger and James 1954). The free energy of the system is given by

$$
\begin{aligned}
& \frac{F}{N k_{B} T}=(z-1) \ln \left[\int g^{z}\left(\theta_{j}\right) d\left(\cos \theta_{j}\right)\right] \\
& -\frac{z}{2} \ln \left[\iiint g^{z-1}\left(\theta_{i}\right) g^{z-1}\left(\theta_{j}\right) f\left(\theta_{i j}\right) d\left(\cos \theta_{i}\right) d\left(\cos \theta_{j}\right) d\left(\phi_{i}-\phi_{j}\right)\right]
\end{aligned}
$$


Assuming that

$$
\begin{aligned}
& E\left(\theta_{i j}\right)=-B^{*} P_{2}\left(\cos \theta_{i j}\right) \text { and } \\
& V\left(\theta_{j}\right)=-B P_{2}\left(\cos \theta_{j}\right)
\end{aligned}
$$

it is not possible to accurately satisfy Chang's consistency condition at all angles. Using a weaker approximation (Ypma and Vertogen 1976) that only the average value of $P_{2}(\cos \theta)$ is the same for the central and outer molecules is equivalent to the BPW approximation of magnetism (see, for instance, Smart 1966) and does not truly minimise the free energy. Taking into account higher order terms in $V\left(\theta_{j}\right)$, Chang's relation can be satisfied quite accurately (Madhusudana et al 1977). The lower the value of $z$, the better the results agree with experiments, and for $z=3$, $T_{N I}-T^{*} \simeq 10^{\circ}$.

Many compounds with very strongly polar groups attached along their long axes also exhibit the nematic phase (see figure 2). What is the role of such dipoles in stabilizing the nematic phase? We postulated some years ago (Madhusudana and Chandrasekhar 1973b) that neighbouring molecules in such cases should have an antiparallel correlation. But since the nematic liquid crystal is a fluid, there is no antiferroelectric long range order. We can rewrite the above theory with

$$
E\left(\theta_{i j}\right)=A^{*} P_{1}\left(\cos \theta_{i j}\right)-B^{*} P_{2}\left(\cos \theta_{i j}\right),
$$

and retain

$$
V\left(\theta_{j}\right)=-B P_{2}\left(\cos \theta_{j}\right)-C P_{4}\left(\cos \theta_{j}\right)-\cdots
$$

The calculations explain the experimental observation of an increase in the average dielectric constant on going from the nematic to the isotropic phase of strongly polar compounds (Schadt 1972; Ratna et al 1973; Ratna and Shashidhar 1977) as arising from a decrease in the antiparallel short-range order between the molecules. Supporting evidence for such a correlation has been obtained recently from X-ray and neutron scattering studies (Leadbetter et al 1979). A consequence of this type of near neighbour antiparallel ordering is the formation of a bilayer structure in such systems, and it turns out that such a bilayer structure plays a key role in the interesting phenomenon of the re-entrant nematic phase which is stable at temperatures below those of the smectic A phase (Cladis 1975, 1980).

\section{Hard particle models}

The shape anisotropy of the nematogenic compounds has not been taken into account in the theories described above. It is known from the theory of liquids made up of spherical molecules that the structure of the fluid is essentially determined by the excluded volume effects (see for instance Barker and Henderson 1976). Several models of the nematic phase have been developed by assuming that it consists of hard rods without any attractive potential between them (see Straley 1973). The properties of such an 'athermal' system depends entirely on its density. 
Onsagar (1949) developed the first theory of phase transition in a system of hard rods. He demonstrated that as the density is increased, such a system undergoes a first order transition from an isotropic phase to an orientationally ordered phase.

Consider a system of $N$ rods in a volume $V$ and temperature $T$. Let the position of the $j$ th rod be $\vec{R}_{j}$ and its orientation $\vec{\Omega}_{j}$. Assuming that $\Omega$ can take only $\nu$ discrete values, we can write the configurational partition function as

$$
Q_{N}=\frac{1}{N !} \frac{1}{\nu^{N}} \sum_{\Omega} \int d \vec{R} \exp \left(-\beta U_{N}\right)
$$

where $U_{N}$ is the potential energy of the system, which can be assumed to be equal to

$$
\begin{aligned}
& \underset{1<i<j<N}{\sum_{i j} \text { and, for hard particles, }} \\
& u_{i j}=\begin{array}{l}
\infty \text { if } i \text { and } j \text { overlap, } \\
0 \text { otherwise. }
\end{array}
\end{aligned}
$$

Performing the integration over the positions only,

$$
\int d \vec{R} \cdot \exp \left(-\beta U_{N}\right)=V^{N} \exp \left[-\beta \phi_{N}(\Omega)\right]
$$

where $\phi_{N}$ is the excess free energy (relative to an ideal gas) of a system of molecules having fixed orientations. If there are $N(1)$ molecules along $\Omega_{1}, N(2)$ along $\Omega_{2}$, etc., we can write

$$
\begin{aligned}
Q_{N} & =\frac{V^{N}}{N ! \nu^{N}} \sum_{N(1)=0}^{N} \cdots \sum_{N(v)=0}^{N} \frac{N !}{\prod_{i=1}^{v} N\left(\Omega_{i}\right) !} \\
& \times \exp \left[-\beta \phi_{N}(N(1) \ldots N(\nu))\right],
\end{aligned}
$$

with

$$
\sum_{i=1}^{v} N\left(\Omega_{i}\right)=N
$$

Defining the mole fractions $x_{i}=N\left(\Omega_{i}\right) / N$, and using the maximum term approximation, the configurational free energy is given by

$$
\frac{\beta}{N} A_{N}=\frac{\beta}{N} \phi_{N}\left[\rho, \tilde{x}_{1} \ldots \tilde{x}_{v}\right]+\sum_{i=1}^{v} \tilde{x}_{i} \ln \tilde{x}_{i}+\ln \nu+\ln \rho-1,
$$

where $\rho=N / V$ is the number density, and the tildes denote that the distribution corresponds to the maximum term of (15). We can now go over to a continuous distribution of angles replacing $x_{i}$ by $f\left(\Omega_{i}\right) . A_{N}$ can then be minimised with reference to $f(\Omega)$ if we can evaluate $\phi_{N}$. 
Onsager made a virial expansion of $\phi_{N}$ and retained only the second virial coefficient. In this case

$$
\phi_{N}=\frac{p}{2} \sum_{i} \sum_{j} x_{i} x_{j} V\left(\Omega_{i j}\right),
$$

where $V\left(\Omega_{i j}\right)$ is the mutual exclusion volume of two rods oriented at an angle of $\Omega_{i j}$. For spherocylinders of radius $a$, cylindrical length $l$ and volume $v_{0}$,

$$
V\left(\Omega_{i j}\right)=8 v_{0}+4 a l^{2}\left|\sin \left(\Omega_{i j}\right)\right|
$$

Onsager's analysis is valid only for low densities and thus for very long rods, with length to breadth ratio $x=(l+2 a) / 2 a \sim 100$. Zwanzig (1963) could calculate higher virial coefficients by restricting the molecules to take only three mutually perpendicular orientations. Flory (1956; Flory and Ronca 1979a) used a lattice model and was able to make calculations at relatively high densities. However, these descriptions are useful only in describing N-I transitions in long polymeric molecules, and the predicted order parameter at the transition is $\sim 0.85$. Further, Saupe (1979) has recently pointed out that the cluster integrals cannot be assumed to be volume independent, and inclusion of volume dependence leads to the result that orientational order may not exist when only a limited number of these integrals are different from zero.

In any case, we are interested in relatively short rods (of $x \sim 3$ to 5) and high densities, and for such systems the scaled particle theory (SPT) provides a convenient method for calculating $\phi_{N}$. SPT was originally developed for a system of hard spheres (Reiss et al 1959) and leads to very satisfactory results in that case. It has been extended to a system of hard rods by many workers (Cotter and Martire 1970; Lasher 1970; Timling 1974; Cotter 1977a). The treatment due to Cotter (1977a) is the most complete and will be summarized below.

We evaluate $W_{i}(\alpha, \lambda, \rho)$, the reversible work of adding a scaled spherocylinder of radius $\alpha a$, cylindrical length $\lambda l$ oriented along $\Omega_{i}$, at any point in the medium. The Gibbs free energy is given by

$$
\frac{\beta G_{c}}{N}=\Sigma x_{i}\left[\ln \left(x_{i} \rho\right)+\beta W_{i}(1,1, \rho)\right] \text {. }
$$

When both $\alpha, \lambda \rightarrow 0$,

$$
a_{i}=\exp \left(-\beta W_{i}\right) \simeq 1-\rho \sum x_{i} V_{i j}(\alpha, \lambda)
$$

where $a_{i}$ is the activity of the $i$ th component, $V_{i j}(\alpha, \lambda)$ the volume excluded to an unscaled molecule $j$ by the scaled particle $i$. When $\alpha$ and $\lambda$ are very large, $W_{i}(\alpha, \lambda)$ approaches the PV work to create a macroscopic cavity in the fluid:

$$
\lim _{\substack{a \rightarrow \infty \\ \lambda \rightarrow \infty}} W_{i}=\left[\pi(\alpha a)^{2} \lambda l+\frac{4 \pi}{3}(\alpha a)^{3}\right] P
$$


It is now assumed that $W_{i}(1,1)$ can be calculated by interpolating between these limits, and standard thermodynamics along with (19) is now used to complete the theory. The phase transition is located by equating the Gibbs free energy and the pressure of the ordered and disordered phases. Calculations show that lower the value of $x$, the density at the transition is higher, while the order parameter and volume change are lower, in qualitative agreement with observed results. For example, $x=2.5$ gives a reasonable agreement with results on PAA (Cotter 1979; Savithramma and Madhusudana 1980a).

How good is SPT in predicting the properties of the hard spherocylinder system? We can compare the equation of state predicted by SPT in the isotropic phase with the results of computer simulation studies on such systems. Monte-Carlo and molecular dynamics studies have been made on systems with $x=2$ and $x=3$ (VieillardBaron 1974; Rebertus and Sando 1977; Nezbeda and Boublik 1978). As the density approaches the value at the phase transition, the calculations become more and more time consuming and impractical. A comparison with the available data shows that while SPT gives reasonably good values at low densities, it overestimates the pressure as the density is increased, and further, the discrepancy also increases at higher densities. Consequently, we can expect that in the nematic state, SPT results are not likely to be accurate.

It would be interesting to extrapolate the essentially 'exact' results of the computer studies to the region of the N-I transition. We found that a model proposed by Andrews (1975) for calculating the equation of state of an assembly of hard spheres can be extended to the case of spherocylinders and used for the extrapolation of the results of computer studies.

Assuming that the introduction of a particle oriented along $\Omega_{i}$ to a system of hard spherocylinders does not change the equilibrium structure of the system, the reciprocal of the 'activity' $a_{i}$ defined in (21) is merely the probability of being able to insert the spherocylinder without overlapping with any other spherocylinder. We can write this as a product of two terms. The first is the probability that an arbitrarily chosen point $r_{N+1}$ does not lie within the core of any of the $N$ molecules. This is given by

$$
\left(1-v_{0} \rho\right)=1-\left(\frac{4}{3} \pi a^{3}+\pi a^{2} l\right) \rho .
$$

Finding such a point ensures that there is no spherocylinder whose centre lies within a volume $v_{0}$ around that point. The second part in calculating $a_{i}^{-1}$ consists of finding the probability, conditional on the first, that the hole around the given point can actually accommodate the introduced particle. This means that the centre of any other spherocylinder, say of the $k$ th type should not lie within the excluded volume of the introduced particle, i.e., within the additional volume

$$
V_{\text {add }}^{i k}=8 v_{0}+4 a l^{2}\left|\sin \Omega_{i j}\right|-v_{0} .
$$

Hence, the probability $P_{k}$ that all $N_{k}$ molecules lie outside $V_{\text {add }}^{i k}$ is given by

$$
P_{k}=\left(1-\frac{V_{\text {add }}^{i k}}{V-\sum_{k=1}^{v} N_{k} \omega}\right)^{N_{k}} \rightarrow \exp \left[-\frac{V_{\text {add }}^{i k} N_{k}}{V-\sum_{k=1}^{v} N_{k} \omega}\right]
$$


where $\left(V-\Sigma N_{k} \omega\right)$ is the 'free' volume available to the molecules. $\omega$ can be expected to be of the order of the average volume occupied by a molecule in a close-packed arrangement. Following Andrews, we can assume that $\omega$ is actually dependent on density and expressible as

$$
\omega=\sum_{n} \omega_{n} \rho^{n}
$$

The reciprocal activity can now be written as

$$
a_{i}^{-1}=\left(1-v_{0} \rho\right) \exp \left[1-\frac{\rho \sum_{k=1}^{v} x_{k} V_{\mathrm{add}}^{i k}}{1-\omega \rho}\right] \text {. }
$$

The thermodynamic properties. of the system can be evaluated using (20). We have made calculations by restricting the expansion in (26) to $n=6$ (Savithramma and Madhusudana 1980a), and determining $\omega_{n}$ by the density of the close-packed solid and six virial coefficients of the system as determined by computer simulation studies. Compared to the SPT calculations, the Andrews model leads to a better agreement with the experimental results on PAA (for example, $x \simeq 3$ ).

We have recently studied the SPT of a system of right circular cylinders (Savithramma and Madhusudana 1980b). The advantage of this system is that it can be used to describe both disc-like molecules (for which the height to diameter ratio is $<0.5$ ) and rod-like molecules (for which the ratio is $>0.5$ ). The thermodynamic properties are symmetrical for the disc-like and rod-like regions if they are plotted as functions of the ratio (largest dimension/shortest dimension) of the cylinders.

Of course, a hard-particle system is not an entirely realistic representation of nematic liquid crystals: a relatively long-range attractive potential is necessary to hold the system together, and to account for the density of the system. Further, defining $\gamma$ as

$$
\gamma=[\partial \ln T / \partial \ln \rho]_{S=\text { const. }},
$$

it is a measure of the relative importance of the volume compared to that of temperature in-determining the variation of $s$ of the medium near $T_{N I}$. Experiments on PAA (McColl and Shih 1972) yield $\gamma=4$. Since hard particle systems are athermal all such models lead to $\gamma=\infty$. On the other hand, mean field models which make use of only an attractive potential yield a $\gamma$ which is equal to the exponent of $V$ in (2). Cotter (1977b) has proved that this exponent can be 1, and only 1 for the mean field theory to be thermodynamically consistent; i.e., $\gamma=1$ in all such models. Hence, both the hard-rod features of the molecules and an attractive potential between them have to be used in a proper description of the nematic phase.

\section{Hybrid models of nematic liquid crystals}

Amongst the many attempts to incorporate both the repulsive and attractive parts of interaction between the molecules, we mention calculations based on the Pople- 
Karasz model (Chandrasekhar et al 1970) which describes both positional and orientational order in terms of a lattice model with a few allowed orientations of the molecules, incorporation of an attractive potential in the Onsager theory (Kimura 1974), and lattice models (Flory and Ronca 1979b) and other models discussed by Alben (1971). Also, higher order terms like $P_{4}(\cos \theta)$ in the mean field potential of Maier-Saupe type theories can be considered to arise from repulsive interactions (Chandrasekhar and Madhusudana 1971; Gelbart and Gelbart 1977).

More recently, an equation of state for the Maier-Saupe model has been derived by assuming the Percus-Yevick approximation for spherical molecules, which arc subject to the attractive potential given by equation (2) (Ypma and Vertogen 1977). Similar studies have also been made using the BBGKY approximation (Lee and Woo 1977). But these models lead to $\gamma \simeq 1$. The anisotropic shape of the molecules has to be taken into account to get higher values of $\gamma$.

Cotter (1977a) used the fact that the activity of the $i$ th species of molecules which are subject to a mean field attractive potential can be written as

$$
a_{i}=a_{i}^{*} \exp \left[u_{i} / k_{B} T\right]
$$

where $a_{i}$ is the activity in the absence of attractive interactions. Assuming that

$$
u_{i}=-\nu_{0} \rho-\nu_{2} \rho s P_{2}\left(\cos \theta_{i}\right),
$$

the SPT was used by her in developing a hybrid model. The resulting distribution function is essentially similar to the Maier-Saupe distribution function, except that the coefficient of the potential has the form $\left[\frac{\nu_{2} \rho}{k_{B} T}+\Lambda(\rho)\right]$, i.e., a temperature-dependent attractive part and an 'athermal' part which has been derived from SPT. For $x=3$ she found that $\gamma \simeq 4$ if the packing fraction $v_{0} \rho \simeq 0.445$, while the experimental value for PAA is $\simeq 0.62$. We (Savithramma and Madhusudana 1980a) have made detailed calculations for other values of $x$ and found that for $x=1 \cdot 75$, the calculated values of many properties are comparable to those of PAA (see table 1 ). We have also used the Andrews model to evaluate $\Lambda(\rho)$ and found that $x \simeq 2.075$ now gives good results (table 1), so that the value of $x$ is now close to the experimental number. Further, the second derivatives of the thermodynamic potential like specific heat $\left(C_{p} / N k_{B} T\right)$, coefficient of thermal expansion $(\alpha)$, compressibility $(\beta)$ are in better agreement with experimental data than those obtained from the SPT. However, the heat of transition $\left(\Delta U / N \dot{k}_{B} T\right)$, volume change $(\Delta \rho / \rho)$, etc. are even now somewhat higher than the experimental values. Partly, this may be the result of the mean field approximation. The resuits may be expected to improve if short range order effects are taken into account.

There have also been a few attempts to derive a generalised van der Waals (GVDW) theory of nematic liquid crystals (Alben 1971; Gelbart and Baron 1977; Cotter 1977c). In this approach, the repulsive part is derived using say the SPT as described earlier but the average attractive energy is derived in the following fashion:

$$
U_{N}=\frac{1}{2 V} \sum_{i} \sum_{j} \tilde{N}_{i} \tilde{N}_{j} \int d r g_{i j}^{(2)}(r) U^{a}\left(r, \Omega_{i}, \Omega_{j}\right)
$$


Table 1. Results of hybrid models with the scaled particle theory and the Andrews model at $T_{N I}$ compared with experimental data on PAA.

\begin{tabular}{lrrr}
\hline$x$ & $\begin{array}{c}\text { Experimental values } \\
\text { on PAA }\end{array}$ & $\begin{array}{c}\text { SPT* } \\
T_{N I}\left({ }^{\circ} \mathrm{K}\right)\end{array}$ & $\begin{array}{c}\text { Andrews } \\
\text { model* }\end{array}$ \\
$s$ & 409 & 1.75 & 2.075 \\
& 0.36 & 409 & 409 \\
$\left(V_{0} \rho\right)$ nen & 0.62 & 0.455 & 0.455 \\
$\Delta \rho / \rho$ & 0.0035 & 0.62 & 0.62 \\
$\gamma$ & 4 & 0.0057 & 0.0062 \\
$\Delta U / N k_{B} T$ & 0.17 & 3.94 & 4.02 \\
$C_{p} / N k_{B}$ & 72.9 (at $\left.T_{c}-1\right)$ & 10.4 & 21.5 \\
$\alpha \times 10^{4} /{ }^{\circ} \mathrm{K}$ & 12.65 (at $\left.T_{c}-1\right)$ & 3.87 & 9.38 \\
$\beta \times 10^{12} \mathrm{~cm}^{2} /$ dyne & 82.3 (at $\left.T_{c}-1\right)$ & 20.5 & 49.63 \\
\hline
\end{tabular}

* from Savithramma and Madhusudana (1980a)

where $g_{i j}^{(2)}(r)$ is the pair correlation function between two molecules with fixed orientations $\boldsymbol{\Omega}_{i}$ and $\boldsymbol{\Omega}_{j}$ and is taken to be of the form

$$
g_{i j}^{(2)}(r) \simeq \exp \left[-\beta U_{i j}^{\star}\left(r, \Omega_{i}, \Omega_{j}\right)\right]
$$

where $U_{i j}^{*}$ is the repulsive potential given by (13). Taking the attractive potential $U^{a}$ to be a sum of isotropic ( $U_{\text {iso }}^{a}$ ) and angle-dependent ( $U_{\text {aniso }}^{a}$ ) parts, Gelbart and Gelbart (1977) have shown that the main contribution to the angular dependent attractive energy arises from the coupling between the $U_{\text {iso }}^{a}$ and the hard rod exclusions. The results are comparable to those given by the SPT with a superposed attractive potential described earlier.

In all these calculations, the molecules are assumed to have cylindrical symmetry. But most real nematogens (see figure 2) have lower symmetry, and Alben (1973) showed that such deviations can account for a lowering of the order parameter at $T_{N I}$. Recent calculations by various authors (Straley 1974; Luckhurst et al 1975; Gelbart and Barboy 1979) have indeed tended to confirm the importance of taking into account the deviations from cylindrical symmetry.

\section{Acknowledgement}

The author's thanks are due to Professor S Chandrasekhar for several useful suggestions. 


\section{References}

Alben R 1971 Mol. Cryst. Liquid Cryst. 13193

Alben R 1973 Phys. Rev. Lett. 30778

Andrews F C 1975 J. Chem. Phys. 62272

Barker J A and Henderson D 1976 Rev. Mod. Phys. 48587

Born M 1916 Sitz. d. Phys.-Math. 25614

Chandrasekhar S 1977 Liquid crystals (Cambridge: Cambridge University Press) Chapter I.

Chandrasekhar S and Madhusudana N V 1971 Acta Crystallogr. A27 303

Chandrasekhar S and Madhusudana N V 1978 in Progress in liquid physics (ed.) C A Croxton (John Wiley) p. 539

Chandrasekhar S, Sadashiva B K and Suresh K A 1977 Pramana 9471

Chandrasekhar S, Shashidhar R and Tara N 1970 Mol. Cryst. Liquid Cryst. 10337

Chang T S 1937 Proc. Camb. Philos. Soc. 33524

Cladis P E 1975 Phys. Rev. Lett. 3548

Cladis P E 1980 in Liquid crystals, Proceedings of an International Conference, Bangalore, December 1979, (ed) S Chandrasekhar (London: Heyden) p. 105.

Cotter M A 1977a J. Chem. Phys. 661098

Cotter M A 1977b Mol. Cryst. Liquid Cryst. 39173

Cotter M A 1977c J. Chem. Phys. 664710

Cotter M A 1979 in Molecular physics of liquid Crystals, (eds) G R Luckhurst and G W Gray

(London: Academic Press) p. 169.

Cotter M A and Martire D E 1970 J. Chem. Phys. 521909

de Gennes P G 1971 Mol. Cryst. Liquid Cryst. 12193

Destrade C, Bernaud M C, Gasparoux H, Levelut A M and Tinh N H 1980 Liquid crystals, Proceedings of International Conference, Bangalore, December 1979, (ed) S Chandrasekhar (London: Heyden) p. 29.

Flory P J 1956 Proc. R. Soc. (London) A234 73

Flory P J and Ronca G 1979a Mol. Cryst. Liquid Cryst. 54289

Flory P J and Ronca G 1979b Mol. Cryst. Liquid Cryst. 54311

Gelbart W M and Barboy B 1979 Mol. Cryst. Liquid Cryst. 55209

Gelbart W M and Baron B A 1977 J. Chem. Phys. 66207

Gelbart W M and Gelbart A 1977 Mol. Phys. 331387

Karat P P and Madhusudana N V 1976 Mol. Cryst. Liquid Cryst. 3651

Kimura H 1974 J. Phys. Soc. Jpn. 361280

Krieger T J and James H M 1954 J. Chem. Phys. 22796

Lasher G 1970 J. Chem. Phys. 534141

Leadbetter A J, Frost J C, Gaughan J P, Gray G W and Moseley A 1979 J. de. Phys. 40375

Lee M A and Woo C W 1977 Phys. Rev. A16 750

Luckhurst G R, Zannoni C, Nordio P G and Segre C 1975 Mol. Phys. 301345

Madhusudana N V and Chandrasekhar S 1973a Solid State Commun. 13377

Madhusudana N V and Chandrasekhar S 1973b Liquid Crystals, Proc. Int. Conf. Bangalore Pramana Suppl. (Bangalore: Indian Acad. Sci.) p. 57

Madhusudana N V, Savithramma K L and Chandrasekhar S 1977 Pramana 822

Maier W and Saupe A 1959 Z. Naturforsch. 14a 882

McColl P R and Shih C S 1972 Phys. Rev. Lett. 2985

Nezbeda I and Boublik T 1978 Czech. J. Phys. B28 353

Onsager L 1949 Ann. N. Y. Acad. Sci. 51627

Ratna B R, Vijaya M S, Shashidhar R and Sadashiva B K 1973 Liquid Crystals, Proc. Int. Conf.,

Bangalore Pramana Suppl. (Bangalore: Indian Acad. Sci.) p. 69

Ratna B R and Shashidhar R 1977 Mol. Cryst. Liquid Cryst. 42113

Rebertus D W and Sando K M 1977 J. Chem. Phys. 672585

Reiss H. Frisch H L and Lebowitz J L 1959 J. Chem. Phys. 31369

Savithramma K L and Madhusudana N V 1980a Mol. Cryst. Liquid Cryst. 6263

Savithramma K L and Madhusudana N V 1980 b Presented at the Eighth International Conference on Liquid Crystals, June 1980, Kyoto, Japan Mol. Cryst, Liquid Cryst. (in Press)

Saupe A 1979 J. de Phys. 40 C3-207 
Schadt M 1972 J. Chem. Phys. 561494

Smart J S 1966 Effective field theories of magnetism (Philadelphia: Saunders)

Straley J P 1973 Mol. Cryst. Liquid Cryst. 22333

Straley J P 1974 Phys. Rev. A10 1881

Timling K M 1974 J. Chem. Phys. 61465

Vieillard-Baron J 1974 Mol. Phys. 28809

Ypma J G Y and Vertogen G 1976 Solid State Commun. 18475

Ypma J G Y and Vertogen G 1977 Phys. Lett. A60 212

Zwanzig R $1963 \mathrm{~J}$. Chem. Phys. 391714 